\title{
METABOLISMO SOCIAL Y DERECHO AMBIENTAL: OTRA MIRADA SOBRE EL ORDEN SOCIAL Y LA NATURALEZA.
}

\author{
REFLEXIONES DESDE LA PERSPECTIVA DE LA SOCIOLOGÍA. ${ }^{1}$
}

\section{Pedro F. HERNÁNDEZ ORNELAS ${ }^{2}$}

\section{Resumen}

Se trata de un ensayo que nace del examen de las relaciones sociales entre seres humanos y los otros elementos de la naturaleza y de la hermandad que de ello resulta por intercambio de energía-materia ( $\left.\mathrm{E}^{\prime}\right)$ física, llamado propiamente "metabolismo social"; visto todo ante un horizonte de sistemas complejos e interdisciplinaridad (científica). Esa base física radical de hermandad, considerada más a fondo de manera fenomenológica, permite hablar, ulteriormente, de una verdadera adscripción y posesión -analogada- de "derecho de la naturaleza"; distinto de los llamados 'derechos naturales '. Alterar las estructuras esenciales de los elementos naturales, es violar derechos. Justificarlo, requiere de un riguroso examen moral.

Palabras clave; Derecho de Naturaleza, Metabolismo-social, Responsabilidad moral, Complejidad inercial de la materia.

\footnotetext{
${ }^{1}$ Este trabajo es acerca de una aproximación a las bases metafísicas del derecho ambiental (derecho de todos los seres de la naturaleza), sus categorías y sus consecuencias elementales (ética y sistemas jurídicos-derecho positivo). El camino aquí propuesto de la fenomenología: análisis crítico de la sencilla presentación del objeto ante el ser humano y sus condiciones de "conocer". La inspiración de esa ruta es una deuda con Heidegger.

2 Profesor-Investigador en el Posgrado de Sociología del Instituto de Ciencias Sociales y Humanidades "Alfonso Vélez Pliego" de la Benemérita Universidad Autónoma de Puebla. Sociólogo especializado en Teoría Social y Humanismo; Ética del Desarrollo Socio- económico; Sociología de la Religión; Ecología y Sociedad-Sociología y Economía Ecológica. pfhernan@prodigy.net.mx.
}

Fecha de recepción del artículo: Julio 2016

Fecha de evaluación: Octubre 2016 


\begin{abstract}
This essay reflects upon the social relations between human actors and other elements of Nature: they become a real brotherhood amongst them, stemming from the mutual exchange of the same matter-energy physical (properly called "social metabolism"). Examined all this against the mirror of complex systems and interdisciplinary assumptions, the radical basis of brotherhood among all elements of Nature reveals the ground upon which the juridical system of "rights of Nature" can be objectively founded, (analogous, delegated rights). More even so, when the basis of all rights is illuminated by phenomenology, a case in point here. Altering essential structures of matter constitutes a violation of rights; justifying it, demands a rigorous examination, moral, of consequences.
\end{abstract}

Key Words: Rights of Nature, Social Metabolism, Human responsibility, Inertial complexity of matter.

\title{
Résumé
}

Il s' agit ici d'une réflexion sur le complexe de relations humaines avec les éléments de la Nature: un tissu qu'aboutit a la vraie fraternité antre eux. La condition, -fraternelleque naît de communication mutuel d'énergie entre eux mêmes on la connais comme "le métabolisme social". On construit l'examen du tout d'àpprés l'hypothèse de des "systèmes complexes", et sur un chemin (scientifique) interdisciplinaire. La fondement physique de la fraternité des êtres de la Terre nous révéle la raison d'être d'un système juridique qui peut définir en rigueur les vrais droits (analogues) de la Nature: tant mieux si l'on examine tout dans une perspective phénoménologique, - le cas présente. L'altération grave des structures d'un élément de la Nature c'est une violation du droit; sa justification dèmand une considération morale sérieuse et objective. de domaines de confrontation

Mots-Clés: Droit de la Nature, Métabolisme Social, Responsabilité humaine, Complexification de la matière. 


\section{Introducción}

La Sociología es la ciencia de las relaciones sociales. Una posible explicación objetiva de la naturaleza de la interacción de toda persona - en grupo o en comunidad — con "su mundo": relaciones con nuestros semejantes y con los demás seres del cosmos. Son ellas, en su compleja totalidad, el gestor de la historia. Pero no es posible olvidar que poseemos solamente una historia: "la historia del hombre es la historia de la naturaleza" (Marx). Esas relaciones se originan en un encuentro: el agente humano, el "otro". Insondable la profundidad de ese simple evento: la mera presentación de la calidad o intimidad del objeto revela al humano su dignidad de "ser-así', y también su posible complementariedad —o sencillamente, utilidad — ante la expectación y anhelo del sujeto humano. Un misterio (la unidad de dos seres) en el evento más simple de la vida cotidiana en toda sociedad, y con él también, la revelación del espacio y del tiempo (Heidegger, Ser y Tiempo, 1959, c. VI).

Esta realidad hace indispensable la renovación continua de los paradigmas y métodos heredados acerca de los conocimientos del ser y de la conducta humana: ir conociendo mejor la verdad de nuestra condición social, la de seres inteligentes y dotados de libertad, hermanados por la misma energía con todos los elementos de la naturaleza que nos abriga y sostiene. La ciencia hoy en día, requiere ampliar la visión del mundo por encima del positivismo mecanicista de sus propulsores (la modernidad), así como del alejamiento del pensar filosófico en muchos investigadores y pensadores actuales de toda ideología. La tradición humanista del conocimiento, en todas las grandes culturas y desde sus mitos fundacionales, nos llevan a la búsqueda continua, humilde, incansable, de nuevas visiones de complejidad en todos los sistemas de energía (estructurada o percibida en caos), y de respeto por el trasfondo misterioso del universo y de la vida... ¡La ciencia no puede separarse de la filosofía porque en ésta última están sus propias raíces!

Sabemos los humanos - lo comprueba la arqueología — que, a diferencia de los otros seres del mundo, nos relacionamos los demás por inercias; ellas siempre, de algún 
modo reflejo, implican la voluntad y la razón, un propósito libre, que sólo se oculta por tiempo limitado cuando se pierde la experiencia de vida, nuestra conciencia, "nuestro ser en el tiempo" (Marx).

Pero sabemos, también, que participamos de la misma energía de todo otro ser, y esa es la conciencia de nuestra hermandad con los elementos de la naturaleza. Ella impone nuevas consideraciones sobre toda la actividad humana, actividad omnipresente que llamamos cultura. Para el sostenimiento y gradual perfección de los humanos y de "su mundo" entero, la cultura se desarrolla por ancho y largo de siete áreas o territorios de la vida en sociedad; sin exceptuar comunidad o sociedad alguna. Surgen así a lo largo de la historia las tareas o actividades relacionadas con lo Numinoso: las tareas del ser humano ante lo sagrado y el misterio, las que se institucionalizan en las religiones. Las tareas familiares, luego, las que responden a la dinámica de la intimidad afectiva y al anhelo de trascendencia, ante todo, de la trascendencia de la vida, la familia. Junto a ellas, las tareas en la búsqueda del orden y la armonía en el vivir social, la política.

En toda cultura y en la historia de las civilizaciones, son ellas las tareas más grandes del humano, las que en toda sociedad buscan respuesta a sus mayores preguntas: ¿qué soy y quién soy?, ¿de dónde vengo, a dónde voy?, ¿qué sentido tiene la vida y qué sentido el dejar de vivir; qué tiene el universo?, etc.

A su lado, cuatro tareas más complementan la actividad humana y conforman así las diversas culturas con rasgos peculiares: tareas de educación, transmisión de los conocimientos necesarios para vivir en los distintos "eco-sistemas" de cada sociedad; tareas de economía, para usar los recursos naturales, universalmente destinados a todos los seres para seguir existiendo; tareas de higiene o salud, para conservar razonablemente la vida, personal y comunitariamente; y, por último, las tareas de recreación, para gozar de algún modo y en tranquilidad la armonía del de "ser-así" en el cosmos. No hay, literalmente hablando, pueblos incultos; pero sí hay sociedades o comunidades con mayores o mejores y más complejas estructuras de sus tareas culturales, lo que Ortega Gasset propone como civilización (Farb, Humankind, 1978, New York, Houghton, cc 2-13). 
Desde los inicios y a lo largo del tiempo, surgen otras tareas que no se desarrollan uniformemente en todas las culturas, pero que atraviesan los terrenos de muchas otras de esas siete tareas básicas. Entre ellas, sobresalen las tareas de la comunicación y las del derecho; ambas de enorme importancia ante la tragedia actual del desastre ecológico planetario, en buena parte producido por la actividad económica de los humanos, desviada por falsos principios y por ignorancia. De todo ello, en este ensayo sólo hablaremos del derecho de los seres no-racionales de la naturaleza, objetos de derecho pero también agentes (bajo ciego dominio) de su historia; y con el ser humano - y por analogía poseedores de derechos a modo - como causantes de esa situación de inédita gravedad en la existencia del mundo por su amenaza de destrucción de la vida humana... Finalmente, un ataque a la vida, vegetal y animal, a la esencia de "ser-así" de muchísimos elementos, destrucción que resulta criminal porque es daño grave a la existencia entre hermanos de una misma energía; ¿vulneración de los derechos de seres sin voluntad ni razón? Sí.

\section{Comunicación y confraternidad en el origen del derecho}

Recordemos primero ¿cómo se originan las actividades, las tareas del ser humano, de dónde provienen? La actividad del humano, como de todo otro ser en el mundo, es la expresión radical de la inercia de su energía. La física contemporánea enseña que la energía, hasta en las mínimas expresiones del ser (los elementos sub-atómicos) revela su inercia como "lineariedad" peculiar de E': su orientación hacia la complementación de su "ser", o sea a hacia la gradual y mayor compleja estructuración (complexification, fr.) de la materia (Teilhard, 1959: 170; Penrose, R. The Road to Reality, 2006, cc. 24-25).

En los seres humanos la energía que se revela en los instintos de otros vivientes y en la existencia milenaria de las demás estructuras de materia, aparece como energía, sobrepasa las leyes que la física (o regularidades de movimiento) en el resto de la naturaleza. En nosotros, es energía al servicio de algún propósito racional. El humano es hasta ahora, como punta de lanza de la evolución cósmica, el único ser que se pregunta 
por el "sentido" de las cosas, ¡el "por qué” del universo y de sí mismo! ¿Qué nos dice todo eso ante los términos de este ensayo?

Parece objetivamente válido ( humana primera, la que da forma a todas, es la comunicación, la revelación de nuestra condición de "ser social". Es ella la manifestación de la lineariedad de complementación (de "realización" individual y estructural, o comunitaria), en los humanos) de nuestro "ser-así", ser personas. Y de tal reconocimiento — la dignidad de "ser"- de las cosas del universo, su peculiar "ser-así”, emerge el derecho como tarea humana fundamental de toda cultura, compañera de toda otra actividad: descubrir en todo y en todos un "ser-así" que es respeto y es "cuidado del ser" (sorge, al.), como lo ha demostrado luminosamente Heidegger (Ser y Tiempo, 1925, c. VI).

El derecho afecta por sí mismo toda conducta del humano; toda conducta lleva en sí una manifestación y un ejercicio de derechos, porque la conducta es "interacción" con los demás elementos del universo y allí ellos muestran su propio ser, muestran su calidad, su esencia que es dignidad, llana y radicalmente, dignidad de ser. Por ella, su “dignidad de ser" y en ella, todo ser deviene sujeto —o puramente objeto- de derecho. Así conocemos que derecho y comunicación son inseparables.

En toda relación, el humano se une — comunicando — a otro "ser-así" que debe respetar, simplemente por el hecho de "ser", por la hermandad de la misma energía que ambos participan. En nosotros, es la intuición más profunda y primigenia de vida humana, ¡reveladora de la propia conciencia! Como sabemos bien, el derecho se entiende (RAE) del modo más elemental como "la facultad natural del hombre para hacer legítimamente todo lo que conduce a los fines de su vida". No es lugar éste par entrar en las diversas clases de derechos, pero sí hay que decir que su origen está en el ser humano y su sacralidad está en la dignidad de su "ser-así', razonables y libres. Por ello, su ejercicio implica razón y libertad, así lo han reconocido las grandes culturas de la humanidad desde el registro de la historia.

Por su parte, el llamado "metabolismo social", no es otra cosa sino el intercambio de energía entre el ser humano y los otros seres de la naturaleza. 
Científicamente, "el concepto - y el proceso - por el cual se describen y cuantifican los flujos de materia y energía que se intercambian entre conglomerados sociales particulares y concretos y el medio natural —ecosistemas, paisajes, etc._-" (González de Molina et. a., 2011, 62). Eso es, en su raíz, energía comunicada, que comprende cinco funciones principales: la apropiación (de elementos: extracción procesada), transformación, distribución, consumo y excreción. El fenómeno está así mismo, en la base de las tareas económicas del ser humano (Georgescu-Roegen, "The Entropy Law and the Economic Problem", Distinguished Lectures, University of Alabama, No.1, 1971).

Ante el fenómeno, hay que reiterar, diciéndolo sencillamente, que el derecho nace del respeto del humano por toda otra criatura por el simple hecho de ser algo, por su calidad de ser, que nos hermana en la misma energía y en proporción a ella; y que la comunicación entre humanos, así como entre ellos y el resto de la naturaleza, revela ese respeto como la condición fundamental del vivir en sociedad. El mal, cosa o acción mala, no es digno de respeto, porque el "ser", todo ser, es un bien, y el mal es en sí mismo "ausencia de bien", ultraje a ser. Dicho sea de paso, el agente (o agentes) del mal será siempre sujeto de derecho, porque su acto (ausentar el bien) no es lo mismo que "ser-humano".

\section{Conciencia ecológica y derechos de la naturaleza}

Entre otras muchas —como la cibernética y la investigación de los sistemas complejos_-, la economía y la sociología ecológica iluminan la conciencia y el valor de nuestra hermandad con toda la naturaleza. La luz que fue aurora de nueva época - la joven conciencia ecológica - emergió de la literatura (obras como las de Muir, Carson, etc., en Norteamérica, fueron el nutriente del llamado principio Bruntland (ONU, Informe Bruntland,1989)) que podría definirse así: "luchar por el desarrollo social sostenible; pero usando los recursos naturales de modo que las futuras generaciones gocen de las mismas condiciones de ambiente y recursos que nosotros que el pasado nos 
legó". Algo que despierta fuertemente una nueva conciencia generacional y alerta la condición moral de la actividad humana, especialmente en la economía.

Ante ella, es necesario examinar críticamente una seria desviación de carácter y conciencia legal (por ende, de suma relevancia en el destecho), tal vez mejor dicho, conciencia de la legitimidad de la conducta humana ante los elementos naturales. Un anti-valor que luego resulta falsamente legalizado en el derecho positivo de los diversos estados nacionales: leyes de expoliación ilegítima de muchos eco-sistemas. Muchos son los actores responsables de esa tragedia, la que hoy encapsulamos en términos de "cambio climático", casi todos nosotros, los actores de la actualidad y pos-modernidad, con excepciones notables quizá, entre los pueblos que han preservado mejor sus herencias indígenas culturales de respeto religioso a la tierra.

Tragedia desgraciadamente agudizada por las grandes ideologías que a partir de la modernidad han venido controlando prácticamente las mayores formas y corrientes de actividad en el planeta: izquierdas socialistas y derechas capitalistas. Maneras de pensar pretendidamente apoyadas en ideologías de crecimiento económico sin barreras y de consumismo irrefrenable (en la izquierda, con mejor distribución social). Inercias al fin de una ética utilitarista y también de una equivocada interpretación religiosa (¿cristiana?) del papel del ser humano frente al resto de la creación y economía que pone hoy a los humanos ante la amenaza muy grave de su propia extinción.

La repetida sentencia del Génesis, "llenen la tierra y sométanla" (Gen. 1, 28), que traduce el sometimiento como "dominio" en la primitiva usanza legal de los romanos, uti et abuti (lat.), parece justificar tales maneras de pensar, hoy en día prácticamente hegemónicas: legitimación pseudo-cristiana en apoyo de una moral equivocada.

Todo el dominio, en vez del encargo superior de "mayordomía" (en inglés, stewardship) o la "humana gestión" de las cosas del mundo, hacia donde apunta el término del original hebreo de la Biblia... Aunque es cierto que la voz hebrea, rabáh (“pisar fuerte", hb.), conlleva dureza (como es el esfuerzo por remover o apisonar la 
tierra — ¡en la tradición y leyenda de pueblos agrícolas!-), una dureza del trabajo humano que contrasta, en la mente del escriba bíblico, con la facilidad con la que Dios sencillamente crea el mundo con una palabra baráh (hb.) (Brown, 1968,11).

Con ello, en las profundidades del imaginario colectivo y las consecuencias que por él afectan el subconsciente de los humanos, emerge con intensidad, sobre todo en la cultura occidental, el síndrome compulsivo de asesinar al hermano, como una de las fuerza motrices de conducta compulsiva (de base subconsciente) que la psiquiatría considera emanada de "los grandes arquetipos (o guías) del macrocosmos" personal o colectivo. Aquí se trata del mito bíblico renovado de Caín y Abel: el propósito, cada día más explícito, de fratricidio de esos hermanos que son los seres de la naturaleza que nos rodea y que nacieron antes de nosotros.

Somos el hermano fratricida los que ahora estamos cada día más dominados por la pasión de consumir todo, de tener las mejores técnicas de producción para amasar legalmente riquezas y gozar más de la vida, de las comunicaciones más rápidas, a fin de ganar más dinero, para comunicarnos mutuamente más y conversar menos, para gozar más efímeramente mientras terminamos con recursos no-renovables. Y todo ello mientras el planeta Tierra — “nuestra nave espacial en el cosmos" (K., Boulding, 1966) - va empezando a agonizar bajo el peso de las energías negativas de esa entropía que traerá, sin duda, nuevas formas de ser y de vivir, pero a costa, quizá, de la propia existencia humana.

\section{Derecho ambiental hoy}

Pero surge, con el despertar de la conciencia ecológica una pregunta muy relevante hoy en día: ¿son los seres naturales no-humanos verdaderos sujetos de derecho ante la activad humana? Yo pienso que es legítimo (es decir, objetivamente sustentable ante la ciencia), responder que sí. Todos los seres naturales no-humanos, son análogamente sujetos de derecho. No en cuanto seres capaces de exigir por sí mismos el trato correspondiente a su dignidad o calidad de ser. Sí lo son, por otra parte, son vicariamente sujetos de derechos porque la posible desviación (perversidad o abuso) en 
el manejo que los sujetos racionales hagan de la energía que los informa —a ellos, los recursos naturales-, destruye estructuras o modos de ser y de informar, a su vez, la vida de muchos otros seres del cosmos.

Dicho de otro modo, hay en todos los elementos de la naturaleza una calidad de ser que por la comunicación y el metabolismo social se confía a los seres humanos. Ellos, son sus actividades en el mundo, tienen a su vez la capacidad de alterar profundamente muchos eco-sistemas que sostienen la vida de muchísimos otros elementos naturales; dicha capacidad, que se delega cuando el ser racional reconoce la dignidad de otro "ser-así", obliga al agente humano a profundizar en el conocimiento de la dignidad confiada a él y sólo llegar a transformar las estructuras (o el modo de ser en la energía de los elementos) si se conoce la manera en que la energía liberada por la estructura que alteramos llega o no a perjudicar el conjunto de algún eco-sistema y las consecuencias posibles de ello para la existencia del mundo.

Termino con un caso irónico que ilustra bien la propuesta derivada de la magnitud social del metabolismo ecológico: el reconocimiento de los seres naturales como sujetos (vicariamente activos) de derecho ante la acción humana, particularmente, frente a la destrucción ambiental. Para muchos, la ciencia actual debe estar orgullosa de haberse deslindado por completo de los mitos y fábulas del pasado. Deseo exponer un caso que no es nada excepcional frente a esa afirmación.

Si hasta hoy ignoramos radicalmente cómo disponer, sin graves riesgos ambientales, de los desechos nucleares que ahora van asfixiando en silencio algunos territorios del planeta, ¿por qué la ciencia —los científicos más calificados, supuestamente - nos lanzó a procesar belicosa y comercialmente la energía atómica? En una comparación campechana, ¿pondría un rico algún capital para abrir una panadería cuyos procesos de elaboración se consideraran tóxicos y prohibidos en todos los países del mundo?. . ¿ ¿Saben por apoyó y entró la ciencia (y el capital mundial) a la 
comercialización del la energía atómica? Por un mito: ¡"nuestra tecnología del futuro nos resolverá el problema”!

Bravo, brevísimo. Mil gracias.

\section{Referencias al texto (y lecturas complementarias*)}

Sugiriendo autores pertinentes, entre los más dignos de recuerdo*:

Carson, Rachel, The Sense of Wonder, Photographs by Charles Pratt and others, New York, Harper and Row, 1956.

Crèvecoeur, Hector St. John, Letters from an American Farmer, New York, Dutton, 1957 (Orig., 1782).

Dubos, René, Celebrations of Life, New York, McGraw Hill, 1981.

Leopold, Aldo, Sand Country Almanach: With Ensays in Conservation from Round River, New York, Oxford University Press, 1966.

Mackinder, Sir Halford John, Democratic Ideals and Realities, London, Constable \& Co., 1909.

*Muir, John, The Wilderness World of John Muir, Boston, Houghton Mifflin,1954.

Schumacher, E. F., Small is Beatiful: Economics as if People Mattered, New York, Harper Collins, 1989. 


\section{Bibliografía}

Croccker, Thomas D., A short history of environmental and resource economics, en Jeroen, C.M. Van den Bergh, Ed., Handbook of Environmental and Resource Economics, Cheltenham, GL, Edward Elgar Publiching Limited, pp. 32-47, 1999.

Boulding, Kenneth, The economics of coming spaceship Earth, en Georgescu-Roegen, Nicholas, The Entropy Law and the Economic Process, Cambridge, MA, Harvard University Press, 1971.

Heidegger, Martin, Sein und Zeit (Ser y tiempo), Ed. J. Mac Quarrie and Edward Robinson, Harper and Row, New Cork, 1962.

Marx, Carl, (ap.) J. Bellamy-Foster, The Ecologcal Rift, Free Press, 2009.

Maturana, Humberto, Biology of Self-Consciousness, en G. Trautteur, Consciousness: Distinction and Reflection, Neapoli, Bibliopolis, 1995.

Mirowski, Philip, More Heat than Light: Economics as Social Physics, Physics as Nature Economics, Cambridge, Cambridge Univ. Press, 1989.

Prigogine, Yllia, El fin de la Certidumbre, Madrid, Taurus, 1997.

Riffkin, Jeremy, The Access Society, New York, Putman Inc., 2000.

Sagoff, M., The Philosopher as Teacher?, Metaphilosophy, 11, pp. 307-325, Sody, W., Wealth, Virtual Wealth and Debt, New York, Dutton, 1933.

Strickland, Jack, Journal of a Tour of the United States of America, Ed. By J.E. Trickland, The New York. 
Weintraub,E.R, General Equilibrium Análisis, New Cork, Cambridge Univ. Press, 1985. 